\title{
Development of bioadhesive chitosan gels for topical delivery of lidocaine
}

\author{
Jaleh Varshosaz ${ }^{* 1}$, Fariba Jaffari ${ }^{2}$, Sara Karimzadeh ${ }^{1}$
}

${ }^{1}$ Department of Pharmaceutics, School of Pharmacy, Isfahan University of Medical Sciences, Isfahan, Iran.

${ }^{2}$ Department of Pharmacology, School of Pharmacy, Isfahan University of Medical Sciences, Isfahan, Iran.

\begin{abstract}
Lidocaine (LC) is a local anesthetic agent. The aim of this study is prolonging the anesthetic effect of this drug for transdermal delivery. Gels of LC hydrochloride were prepared with three different molecular weights (MW) and concentrations of chitosan. lecithin was used as permeation enhancer. Viscosity, bioadhesion, drug release from synthetic membranes, drug permeation through the biological barrier (rat skin) and antinocicetive effect of gels were studied. Increasing the concentration of chitosan caused to decrease the bioadhesion. Drug release studies in gels showed that increasing the concentration and MW of chitosan caused an increase in both the rate and extent and also in flux of drug probably because of the increase in repulsive forces between LC and chitosan cations. The flux of drug through the rat skin was higher for $3 \%$ high MW chitosan gel $(\mathrm{H} 3)$ compared to the standard gel. LC was effective topically in hind paw formalin assay. It was most active immediately after its administration. The analgesic activity of $\mathrm{LC}$ in $\mathrm{H} 3$ gel could cover the duration of the formalin nociception. The maximal response of $\mathrm{LC}$ in comparable doses of $\mathrm{H} 3$ and standard gel was about $52 \%$ and $36 \%$ analgesia in the second phase, respectively compared to the control group. The higher response of the $\mathrm{H} 3 \mathrm{gel}$ may be attributed to the bioadhesive effect of chitosan base and the higher concentrations of LC compared to the standard gel.
\end{abstract}


Keywords: Lidocaine, Chitosan gel, antinociception, transdermal delivery.

\section{Introduction}

Transdermal and topical delivery of drugs provide advantages over conventional oral administration. The benefit of transdermal systems includes convenience, improved patient compliance and elimination of hepatic first pass effect. Nevertheless, most drugs are not applicable to this mode of administration due to the excellent barrier properties of the skin. Molecules must first penetrate the stratum corneum, the outer horny layer of the skin. The molecule then penetrates the viable epidermis before passing into the papillary dermis and through the capillary walls into systemic circulation. It is the stratum corneum, a complex structure of compact keratinized cell layers that presents the rate limiting step and the greatest barrier to absorption of topical or transdermally administered drugs [1]. LC is frequently used on skin in order to suppress pain from burning, itching, surgical operations, injections and dermatological diseases [2]. During the last three decades, a variety of transdermal anesthetic preparations have been developed; however, these have been documented almost invariably to be ineffective due to insufficient concentrations of the uncharged base (the active form) of the anesthetics or to poor skin absorption secondary to the lipophilic characteristics of amide derivatives (LC, benzocaine, etc.). In trying to increase the anesthetic base concentrations, organic solvents have been used, but unacceptable local irritation prevented their clinical application. Because of their poor penetration through intact skin, these solutions can be used only to anesthetize mucous membranes. Production of oil-in-water emulsion of single anesthetic agents may help in achieving relatively high concentrations of the active form with better skin penetration due to the presence of water. These preparations, however, do not produce effective skin analgesia even with high concentrations attainable [3]. The common method to improve drug permeation through the skin is to use penetration enhancers i.e., organic solvents like ethanol or $N$-methylpyrrolidone, fatty acids like 
oleic acid, surfactants like sodium laurate, ethyltrimethylammonium bromide and lecithin or cyclodextrins [4]. Penetration enhancers can change the structure of skin lipids and alter the skin barrier function. These compounds often generate massive skin irritation [5]. The permeation of LC has been enhanced 1.39-fold in the hydrophilic formulation and between 1.25- and 1.76-fold in the lipophilic formulations by phloretin in the tested vehicles [2]. By passive delivery, transdermal in vitro studies, Kushla and Zatz [6] have shown that cationic surfactants may remarkably increase the flux of LC through human skin from saturated systems in propylene glycol-water mixtures. Kushla et al. [7] investigated the anaesthetic activity of several topical LC formulations containing $20 \%$ propylene glycol. They found that all formulations containing propylene glycol produced significantly greater anesthesia compared with the formulations without propylene glycol. Sarpotdar and Zatz [8] report an in vitro study of the penetration enhancement of LC through hairless mouse skin in the presence of propylene glycol. They observed that the concentration of propylene glycol strongly affects the steady state flux [8]. Lidocaine gels containing HPMC and diethylene glycol showed about 3.89-fold increase in analgesic activity compared with the control [9]. The transdermal delivery of LC by iontophoresis, electroporation, and electroincorporation has resulted in similar surface skin anesthesia; however, iontophoresis results in a greater depth of anesthesia [10]. The results show that if administered by means of gel, the same amount of LC gives significantly higher intracameral levels of LC, better analgesia, better patient cooperation, and less need for intraoperative supplemental anesthesia [11].

Chitosan with excellent biodegradable, biocompatible and bioadhesive properties is a naturally occurring polysaccharide. Due to its unique polymeric cationic character, gel and film forming properties, chitosan has been extensively examined in the pharmaceutical industry for its potential use in the development of drug delivery systems. As there is not any report on the use of chitosan bioadhesive gels for transdermal delivery of LC, the aim of this study was to design a formulation containing chitosan for prolonged local delivery of LC hydrochloride to the skin. Gel 
formulations of chitosan incorporating LC hydrochloride were developed and investigated in vitro for release properties and in vivo anesthetic effects in rat.

\section{Experimental}

\section{Materials}

LC hydrochloride as a gift from Daroupakhsh Pharmaceutical Company (Iran), Chitosan with different molecular weights (MW) (low MW 150000, intermediate MW 400000 , and high MW 600000 , Fluka, Switzerland), formalin $37 \%$ solution, sodium hydroxide, and potassium dihydrogen phosphate were all purchased from Merck Chemical Company (Germany), LC marketed gel ( $2 \mathrm{w} / \mathrm{w} \%)$ was used as the standard gel (Daroupakhsh Pharmaceutical Company, Iran).

\section{Preparation of gels}

Chitosan gels were prepared at $1-3 \mathrm{w} / \mathrm{w} \%$ concentrations in dilute lactic acid solution $(2 \mathrm{w} / \mathrm{w} \%)$. LC was incorporated into the formulations at $4 \mathrm{w} / \mathrm{w} \%$ concentration and $1 \mathrm{w} / \mathrm{w} \%$ of lecithin was added as penetration enhancer. Since the viscosity of the gels increased with the molecular weight, these concentrations were chosen for ease of application. The effect of 2 variables i.e., the molecular weight of chitosan and its concentration each at 3 levels were studied by a full factorial design and $3^{2}$ different formulations were prepared. The formulations are coded with a letter indicating the chitosan MW ( $L$ for Low, $M$ for medium and $\mathrm{H}$ for high MW) and with a number indicating its concentration.

\section{Viscosity}

Rheological experiments were performed to examine the viscous and elastic properties of the different formulations. Viscosity measurements of gels were performed on a Mettler digital non-oscillating, static viscometer (Model RM 180, cup \& bob, Germany) at room temperature. The situation of the test was as follows: 
Measurment sytem No. 33, measuring tube No. 3 diameter $15.18 \mathrm{~mm}$, measuring bob No. 3 diameter $14 \mathrm{~mm}$, shear rate range $6.5-1291 \mathrm{~S}^{-1}$, and fill volume was $9 \mathrm{ml}$.

\section{Bioadhesion measurements}

Bioadhesion was examined in vitro using excised skin of the neonate rat without any further treatment. The maximum force of detachment was measured on a tensile strength tester (Instron, A301, England). Chitosan gels $(0.5 \mathrm{~g})$ were homogeneously spread on a $2.5 \times 2.5 \mathrm{~cm}$ glass disk and then the disks were fixed to the support of the tensile strength tester using a double side adhesive. The gel was brought into contact with the excised skin of the neonate rat under a very slight pressure $(2 \mathrm{~g})$ and was kept in this position for $1 \mathrm{~min}$. Then the tensile test was performed at a constant extension rate of $20 \mathrm{~mm} / \mathrm{min}$.

\section{In vitro release of $L C$}

The release of LC from different chitosan gels was determined by using dialysis cellulose acetate membrane (cut off 12000D, Biogen, Belgium) and the skin permeation through the excised rat skin on a Franz (vertical) diffusion cell. The synthetic or natural membranes were mounted between the receptor and donor compartments (with the stratum corneum side facing upwards). The donor compartment was charged with $0.6 \mathrm{~g}$ of gels. The receptor compartment was filled with $27 \mathrm{ml}$ of Phosphate Buffered Saline (PBS, pH 7.4) which was maintained at $37 \pm 0.5^{\circ} \mathrm{C}$ and stirred by magnetic bar at $200 \pm 5 \mathrm{rpm}$. $\mathrm{LC} \mathrm{HCl}$ is very soluble in this medium. The available diffusion area of cell was $4.91 \mathrm{~cm}^{2}$. At predetermined time intervals (every $0.5 \mathrm{hr}$ until $3 \mathrm{hr}$ and then every $1 \mathrm{hr}$ until $6 \mathrm{hr}$ ), $1 \mathrm{ml}$ sample was taken from the receptor cell and immediately replaced by an equal volume of fresh receptor solution. The samples were filtered, diluted to $20 \mathrm{ml}$ and assayed for LC at $263.3 \mathrm{~nm}$ using a UV spectrophotometer (UV mini 1240, Shimadzu, Japan). Blank chitosan gels were used to determine the probable interaction of gel base with the drug absorbance. In the case of skin permeation test the interferences of uvabsorbing molecules was checked before loading the cell with the gels. 


\section{In vivo anesthetic effect (Paw formalin assay)}

All animal studies were conducted in accordance with the Guide for Care and Use of Laboratory Animals published by the National Institutes of Health and the ethical guidelines of the International Association for the Study of Pain. The formalin test was performed in mice (weight $30-40 \mathrm{~g}$ ) that had been individually exposed to the observation chamber for 45 min before experiments. For the formalin injection, 20 $\mu \mathrm{l} 5 \%$ formalin [12] was administered subcutaneously into the plantar surface of the right hind paw using a 30-gauge needle. The animals were then placed in a clear Plexiglas cylinder $(20 \times 30 \mathrm{~cm})$ for observation. The pain behavior was quantified by determining the amount of time(s) the mouse spent licking the injected paw, over $30 \mathrm{~min}$ using $5 \mathrm{~min}$ bins. Two phases of spontaneous licking behavior were observed after the formalin injection. The interval from 0 to $5 \mathrm{~min}$ has been defined as Phase I, and the interval from 15 to $30 \mathrm{~min}$ has been defined as Phase II [13]. Criteria for exclusion from the study included incomplete formalin injection, or excessive bleeding from the injection site. Time-response data was presented as the mean \pm S.E.M. of $5 \mathrm{~min}$ bins over $30 \mathrm{~min}$. All tested drugs were given $30 \mathrm{~min}$ before formalin administration and were compared to blank chitosan gel administration (negative control group) and the standard marketed LC gel (positive control group). The test gel (H3 that was a $4 \%$ gel) was given $250 \mathrm{mg}$ and the standard gel $500 \mathrm{mg}(2 \% \mathrm{gel})$. Each group consisted of 6 animals. In each case, the cumulative licking response was calculated for each mouse.

\section{Results and Discussion}

An ideal formulation for local delivery should exhibit ease of delivery, a good retention at the application site and a controlled release of the drug. The application of bioadhesive gels provides a long stay, adequate drug penetration, high efficiency and acceptability. Chitosan as a non-toxic, biocompatible and biodegradable polymer, has been widely used for pharmaceutical and medical applications. Chitosan has been shown to promote absorption of small polar molecules and peptide/protein drugs in animal models and human volunteers [14]. Considering the 
enhanced permeability effect of chitosan gels on the hydrophilic molecules through the skin by using lactic acid compared to acetic acid, and less viscosity of the gels produced by this organic acid [15], chitosan dissolved in lactic acid was chosen for gel preparation.

It was seen from the flow curves (Fig. 1) that LC containing chitosan gels exhibit pseudoplastic flow, and viscosity increases significantly with increasing either the chitosan concentration (Fig. 1a) or its MW (Fig. 1b). However, no statistical difference was seen between low and medium MW. Due to the results of bioadhesion measurements (Fig. 2) and high viscosity of its gels (Fig. 1), chitosan is expected to release the drug for a long period of time (Fig. 3), and this enhances the clinical effects. The viscosity of $3 \%$ chitosan gel was found to be higher than that of $1 \%$ and $2 \%$ gels, which makes it more applicable for long retention of drug on topical application but without any difficulty in spreading. The results of viscosity measurements show increasing the shearing stress of the gels by increasing the chitosan concentration and also MW (Fig. 1). However, it seems that chitosan concentration has a more critical effect on the shear stress than the MW as L3 and M3 show similar viscosities (Fig. 1b). 

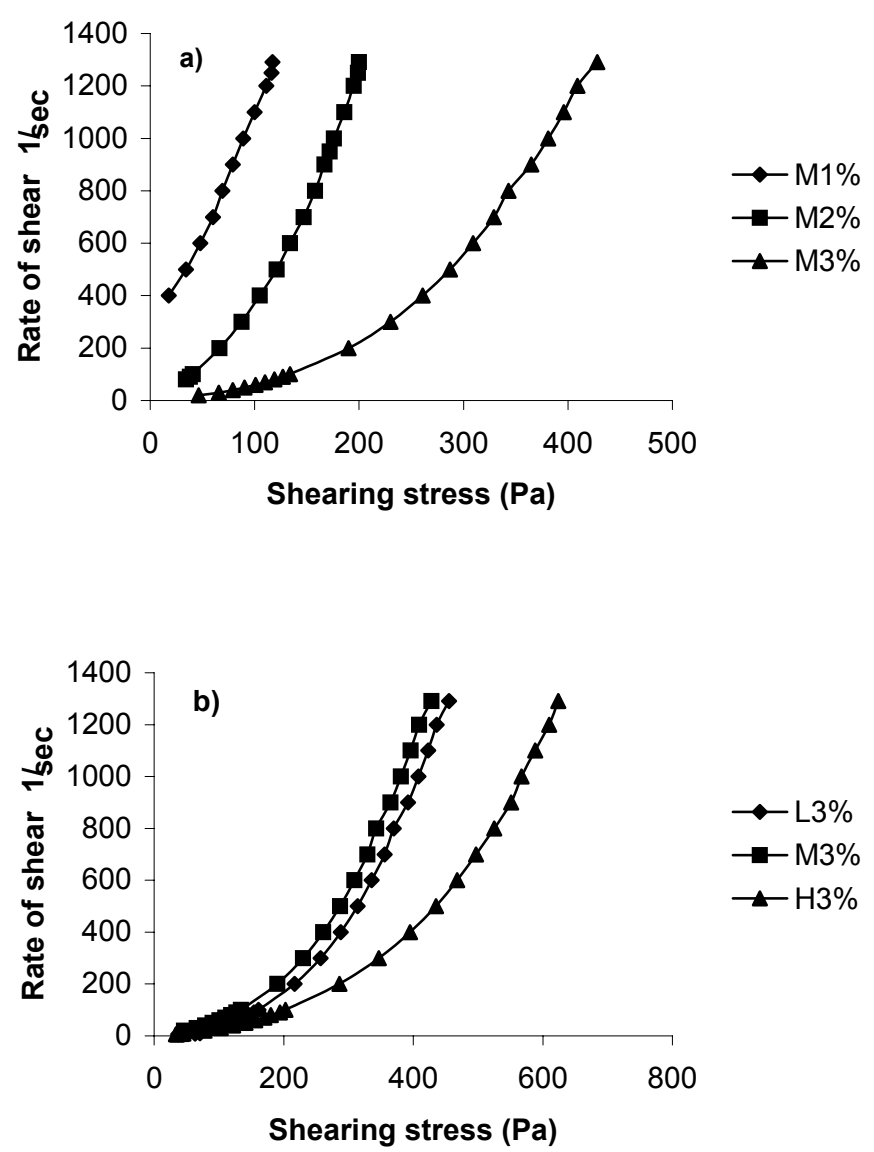

Figure 1: Flow curves of chitosan gels a) with different concentrations of medium molecular weight of chitosan, b) with different molecular weights of $3 \%$ chitosan gels.

The results of bioadhesion measurements are shown in Fig. 2. As this figure indicates regardless of the type of chitosan, increasing the concentration of chitosan decreases the bioadhesion of the gels significantly $(p<0.05)$. No significant difference was found in adhesion force of gels prepared in a specific concentration but with different types of chitosan. 


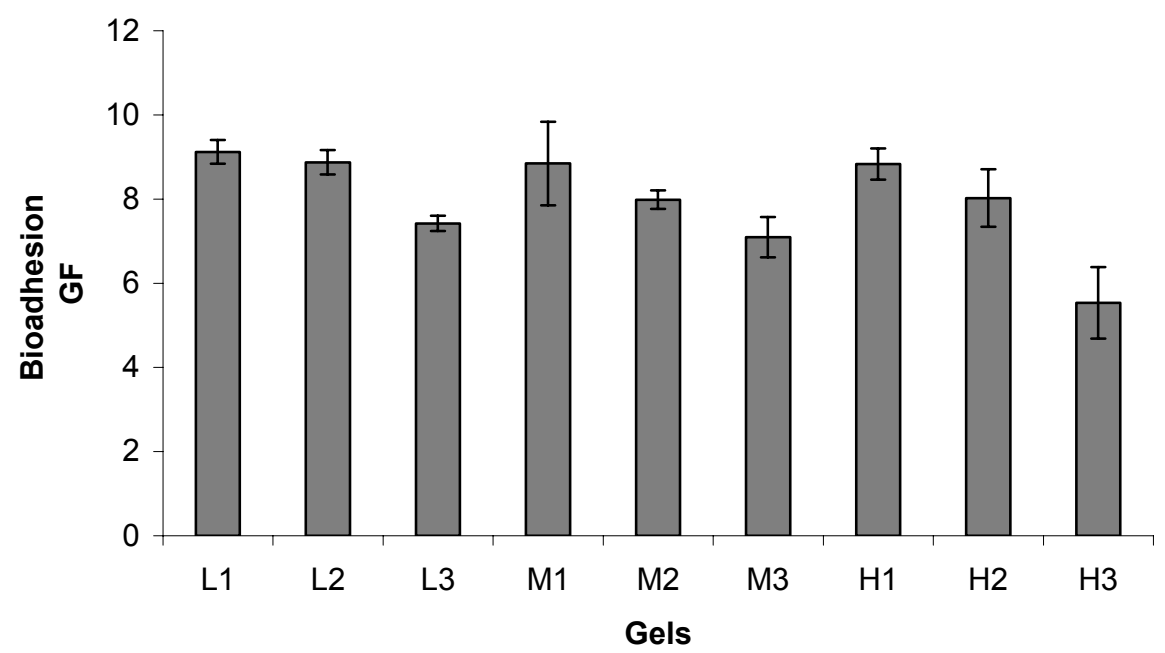

Figure 2: Bioadhesion force (according to Gram force) of chitosan gels ( $n=6$, mean $\pm S D)$.

As bioadhesion measurements of the gels indicate (Fig. 2), increasing the chitosan concentration in each MW, decreases the bioadhesion significantly $(P<0.05)$ but in a constant concentration no significant difference is seen between different MW of chitosan $(P>0.05)$. It may be concluded that by increasing the shearing stress of the gels (Fig. 1), bioadhesion has decreased (Fig. 2). In solid dosage forms, increasing the polymer concentration, promotes the bioadhesion but in the gels, there is a ceiling effect or optimum concentration for the polymer in which at greater concentrations the bioadhesion decreases. This is because of the reduction in the solvent and increased coiling of the polymer chain [16].

LC release profiles through cellulose acetate membrane are seen in Fig. 3. As this figure indicates increasing the chitosan concentration from 1 to $3 \%$ resulted in an increase in LC release (Fig. 3a). This was seen in all MW of chitosan. On the other hand, increasing the MW of chitosan also accelerated the drug release (Fig. 3b). 

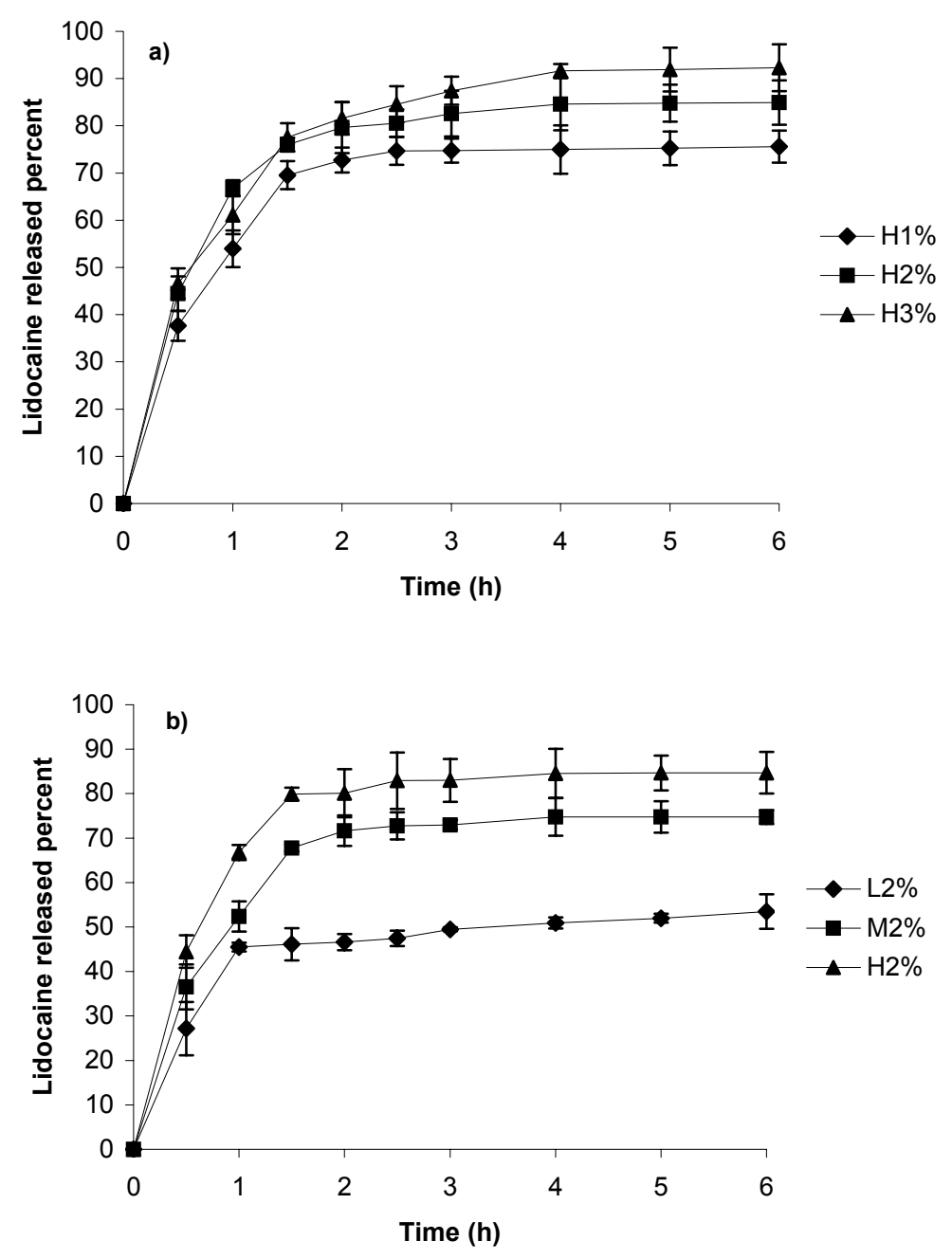

Figure 3: Release of lidocaine $\mathrm{HCl}$ from a) chitosan gels containing different concentrations of high molecular weight of chitosan, b) different molecular weights of $2 \%$ chitosan gels in phosphate buffer solution $(\mathrm{pH} 7.4)(\mathrm{n}=3)$.

The release data reveal that increasing the concentration of chitosan in a particulate MW increases the drug release rate (Fig. 3a) that is not in accordance with viscosity results (Fig. 1a). In other words, the higher the shearing rates of the 
gel, the faster the release of LC. Senel et al. [17] also reported an increase in chlorhexidine gluconate release rate by increasing the chitosan concentration. However, in a specific concentration of chitosan, increasing the MW accelerates the drug release (Fig. 3b). These data suggest the possibility of achieving controlled drug release by the use of chitosan matrices. The direct dependence of LC release on polymer MW is consistent with the increased availability of amino groups for ionic repulsion of the similar charged drug ions. As the molecular weight increases, more amino groups may become available for ionic repulsion of the similar charged drug ions. Rege et al. [18] used chitinosans as tableting excipients for controlling the release of salicylic acid and found at acidic $\mathrm{pH}$ chitinosans had protonated amines which could interact with oppositely charged drug ions and thereby modify drug release.

\begin{tabular}{ccc}
\hline Formulation & $\begin{array}{c}\text { Flux } \\
\boldsymbol{\mu g} / \mathbf{c m}^{\mathbf{2}} \mathbf{h r}\end{array}$ & $\begin{array}{c}\text { Correlation coefficient } \\
\mathbf{r}^{\mathbf{2}}\end{array}$ \\
\hline L1 & $277.45 \pm 8.06$ & $0.93 \pm 0.04$ \\
L2 & $353.48 \pm 11.15$ & $0.91 \pm 0.06$ \\
L3 & $396.22 \pm 9.54$ & $0.93 \pm 0.02$ \\
M1 & $474.00 \pm 12.91$ & $0.93 \pm 0.01$ \\
M2 & $513.10 \pm 4.28$ & $0.92 \pm 0.05$ \\
M3 & $557.56 \pm 4.68$ & $0.93 \pm 0.05$ \\
H1 & $519.93 \pm 24.51$ & $0.93 \pm 0.04$ \\
H2 & $584.65 \pm 30.29$ & $0.94 \pm 0.04$ \\
H3 & $606.89 \pm 19.54$ & $0.93 \pm 0.05$ \\
H3 & $280.05 \pm 30.13$ & $0.97 \pm 0.02$ \\
Standard gel $^{*}$ & $171.13 \pm 38.00$ & $0.92 \pm 0.02$ \\
\hline
\end{tabular}

Table 1: $\quad$ Lidocaine flux from chitosan gels in PBS $(\mathrm{pH} 7.4)$ using cellulose acetate membrane and correlation coefficient of regression analysis of release data according to Higuchi model. (In the cases shown by ${ }^{*}$, flux is determined on excised rat skin) $(n=3)$. 
Ramanathan and Block [19] used iontophoresis for transdermal delivery of different drugs using chitosan gel. They observed the mutual repulsion between LC hydrochloride (as the cationic drug) and the positively charged chitosan matrix caused the chitosan macromolecule to stretch and this could lead to a decrease in the total extent of electro-osmosis of LC towards the anode. These findings explain the faster release of LC from higher concentrations and MWs of chitosan compared to lower concentrations and MWs.

Analysis of release data for different formulations is shown in Table 1. As this table shows LC flux increases by the concentration and MW of chitosan. By increasing the drug concentration in the gels from $2 \%$ in the standard gel to $4 \%$ in $\mathrm{H} 3$ gel, the flux of drug also increases significantly on the excised rat skin. This table also shows that in all cases the Higuchi model describes the release kinetic of LC through gels.

Considering the higher flux of drug through $\mathrm{H} 3$ compared to other formulations, this gel was selected for in vivo anesthetic effect studies using the paw formalin assay test [12]. Kolesnikov et al. [12] showed the effect of formalin administered intradermally into the tail and hind paw of the rats. Following the injection of the formalin, the only behavior suggestive of pain is an intermittent licking of the tail that starts within 2-4 min and lasts $40-60 \mathrm{~min}$. The maximal nociceptive response is observed during the first $30 \mathrm{~min}$, with a subsequent increase of licking activity for the next 30-40 min. Afterwards, the mice dose not show any behavior suggestive of nociception. In the current study, LC was the most active immediately after its administration. The analgesic activity of LC gels could cover the duration of the formalin nociception. Considering the dose adjustment of the $\mathrm{H} 3$ and standard gel Fig. 4 reveals a prompt decrease in licking behavior in standard gel, which gradually increased to control levels, presumably reflecting the shorter duration of the drug effect than the pain as less licking happened in the first phase compared to the second phase. However, the higher LC dose in $\mathrm{H} 3$ gel produced a greater suppression of formalin-induced nociceptive behavior (less licking in both phases) with a longer duration of action (reduction in licking time during the second phase 
compared to the standard gel).In comparable doses applied, the maximal response of $\mathrm{LC}$ was about $52 \%$ and $36 \%$ analgesia in the second phase for $\mathrm{H} 3$ standard and gels, respectively compared to the control group (Fig. 4). The higher response of the $\mathrm{H} 3$ gel may be attributed to the bioadhesive effect of chitosan, prolonged release of LC and higher flux of the drug through the skin. This positive effect of chitosan maybe also caused by opening the tight junctions [20].

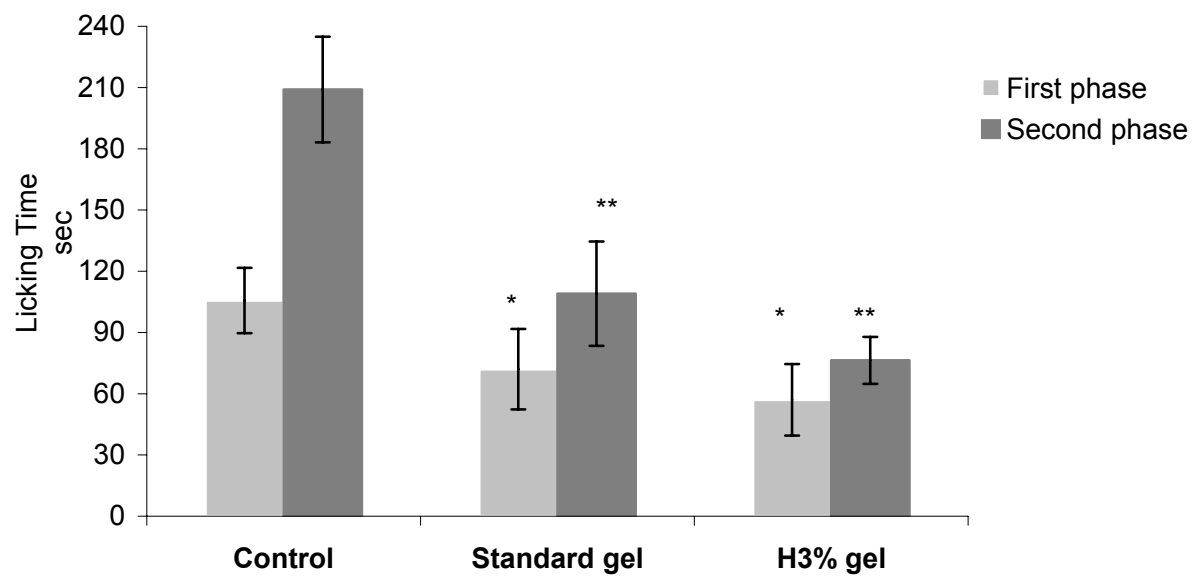

Figure 4: Lidocaine response in formalin paw test. Groups of mices $(n=6)$ received $20 \mu \mathrm{l}$ of $5 \%$ formalin subcutaneously into the paw $30 \mathrm{~min}$ after administration of the gels and the cumulative licking response was calculated for each mouse in two phases; I: 0-5 min and II: 1530 min after administration of $250 \mathrm{mg}$ of $\mathrm{H} 3$ gel containing $4 \%$ drug and $500 \mathrm{mg}$ of $2 \%$ standard gel. ${ }^{*},{ }^{* *}$ means $p<0.05$ in phase I and phase II, respectively compared to the control group.

\section{Conclusion}

It can be concluded that high molecular weight of $3 \%$ chitosan prepared with lactic acid may be promising for local delivery of LC. This gel can release the drug with no lag-time and produce a substantial and longer antinociceptive effect compared to $2 \%$ marketed LC gels. However, further studies with human skin would be interesting. 


\section{References}

[1] Aulton, M E, editor.

Transdermal drug delivery.

In: Pharmaceutics The Science of Dosage Form Design. Second ed. London: Cherchill Livingston, 2002:505- 508.

[2] Auner B G, Valenta C.

Influence of phloretin on the skin permeation of lidocaine from semisolid preparations.

[3] Koren G.

Topical skin anesthesia.

Clin. Dermatol. 1989;7(3):136-141.

[4] Smith E W, Maibach H I, editors.

Penetration enhancer classification.

In: Percutaneous Penetration Enhancers. Boca Raton: CRC Press, 1995:5-20.

[5] Hadgraft J, Guy R H, editors.

Penetration enhancers and their use in transdermal therapeutic systems.

In: Transdermal Drug Delivery. New York: Marcel Dekker, 1989:197246.

[6] Kushla G P, Zatz J L.

Evaluation of a noninvasive method for monitoring percutaneous absorption of lidocaine in vivo.

Pharm. Res. 1990;7:1033-1037.

[7] Kushla G P, Zatz J L, Mills Jr. O H, Berger R S.

Noninvasive assessment of anesthetic activity of topical lidocaine formulations.

J. Pharm. Sci. 1993;82:1118-1122.

[8] Sarpotdar P P, Zatz J L.

Evaluation of penetration enhancement of lidocaine by nonionic surfactants through hairless mouse skin in vitro.

J. Pharm. Sci. 1986;75:176-181.

[9] Shin S C. Cho C W, Yang K H.

Development of lidocaine gels for enhanced local anesthetic action. Int. J. Pharm. 2004;287(1-2):73-78.

[10] Wallace M S, Ridgeway B, Jun E, Schulteis G, Rabussay D, Zhang L.Topical delivery of lidocaine in healthy volunteers by electroporation, electroincorporation, or iontophoresis: An evaluation of skin anesthesia. Reg. Anesth. Pain Med. 2001;26(3):229-238.

[11] Bardocci A, Lofoco G, Perdicaro S, Ciucci F, Manna L. Lidocaine $2 \%$ gel versus lidocaine $4 \%$ unpreserved drops for topical anesthesia in cataract surgery. A randomized controlled trial.

Ophthalmol. 2003;110(1):144-149. 
[12] Kolesnikov Y, Cristea M, Oksman G, Torosjan A, Wilson R.

Evaluation of the tail formalin test in mice as a new model to assess local analgesic effects.

Brain Res. 2004;1029:217-223.

[13] Abbot F V, Franklin K B, Westbrook R F.

The formalin test: scoring properties of the first and second phases of the pain response in rats.

Pain. 1995;60:91-102.

[14] Illum L, Farraj N F, Davis S S.

Chitosan as a novel nasal delivery system for peptide drugs.

Pharm. Res. 1994;11:1186-9.

[15] Orienti I, Luppi B, Zecchi V.

Chitosan and its $\mathrm{N}$-carboxyethyl and $\mathrm{N}$-aminoethyl derivatives as vehicles for topical formulations.

J. Cosmet. Sci. $1999 ; 50: 307-313$.

[16] Ugweke M I, Verbeke N, Kinget R.

The biopharmaceutical aspects of nasal mucoadhesive drug delivery.

J. Pharm. Pharmacel. 2001; 53(1):3-22.

[17] Senel S, Ikinci G, Kas S, Yousefi-Rad A, Sargon M F, Hincal A A.

Chitosan films and hydrogels of chlorhexidine gluconate for oral mucosal delivery.

Int. J. Pharm. 2000;193:197-203.

[18] Rege P R, Shukla D J, Block L H.

Chitinosans as tableting excipients for modified release delivers systems.

Int. J. Pharm. 1999;181(1):49-60.

[19] Ramanatum S, Block L H.

The use of chitosan gels matrices for electrically modulated drug delivery.

J. Control. Rel. 2001;70(1-2):109-123.

[20] Smith J.

Chitosan and transdermal drug delivery.

Retinoids 2003; 19: 72-75. 